\title{
Impact of trimodality treatment on patient quality of life and arm function for superior sulcus tumors
}

\author{
Cornelis G Vos, MD, PhD, ${ }^{a}$ Ramon R Gorter, MD, ${ }^{a}$ Koen J Hartemink, MD, PhD, ${ }^{\text {ab }}$ and J \\ Wolter A Oosterhuis, $\mathrm{MD}, \mathrm{PhD}^{\text {ac }}$
}

${ }^{a}$ Department of Surgery, VU University Medical Center, Amsterdam, the Netherlands; 'bepartment of Surgery, The Netherlands Cancer Institute - Antoni van Leeuwenhoek Hospital, Amsterdam, The Netherlands; and 'Department of Surgery, MCH/Bronovo/ A12 Oncology Center, Den Haag, The Netherlands

Background Trimodality treatment leads to improved survival for superior sulcus tumor (SST) patients. Not much is known about the impact of this treatment on arm function and patient quality of life.

Objective To analyze arm function and quality of life in SST patients undergoing trimodality treatment.

Methods This was a prospective cohort study of consecutive SST patients treated with trimodality treatment that was conducted between April 1, 2010 and October 31, 2012. We obtained informed consent for 20 of 22 eligible patients. The 36-item Short Form Health Survey (SF-36) and disabilities of the arm, shoulder, and hand (DASH) questionnaires were used to asses patient quality of life and subjective arm function at $\mathrm{O}$ (preoperative day), 3, and 12 months after trimodality treatment.

Results DASH scores were significantly lower at 3 and 12 months $(P=.024$ and $P=.011)$ compared with preoperative scores. Significantly lower scores were reported for the SF-36 domains of physical functioning at 12 months $(P=.020)$ and of physical role functioning at 3 months $(P=.041)$, and significantly more pain was reported at 3 and 12 months $(P=.006$ and $P=.019$, respectively). Patients who underwent T1 nerve root resection had lower scores for the SF-36 domain health change at 3 months $(P=.037)$ compared with those in whom the T1 root was spared. For all other domains no differences were found.

Limitations Small sample size; patient prechemoradiation function and quality of life unknown.

Conclusion Subjective arm function and patient quality of life is reduced following trimodality treatment. Resection of the $\mathrm{T} 1$ nerve root has no significant long-term effect on the subjective arm function and quality of life.

0 tandard treatment for superior sulcus tumors (SST) is induction chemoradiotherapy followed by surgery. ${ }^{1}$ This trimodality treatment leads to improved survival in these patients but is associated with considerable morbidity. ${ }^{2}$ SST typically invade the chest wall and possibly the brachial plexus, subclavian vessels, and/or spine. ${ }^{2}$ On the one hand, impaired arm and shoulder function can be caused by direct invasion or compression of the brachial plexus or blood vessels by the tumor. Another cause is the local detrimental effect of radiotherapy and surgery in this region rich of vital structures. In addition, systemic chemotherapy can lead to neuropathy, which could further increase arm and shoulder disability. On the other hand, trimodality treatment could also reduce impairment by reducing invasion or compression.

Not much is known about the exact impact of trimodality treatment on arm function and patient quality of life, ${ }^{3}$ yet knowledge of the impact could be useful in identifying factors that could help optimize perioperative supportive care - for example, intensified physiotherapy for specific functions. Moreover, this improved knowledge could aid pre-operative counseling. Therefore, we prospectively analyzed both arm and shoulder function-related and overall quality of life in a cohort of SST patients before and after trimodality treatment.

\section{Materials and methods \\ Patient selection}

After approval of the VU University Medical Center Medical Ethics Board, all consecutive SST patients between April 1, 2010 and October 31, 2012 were included. SST were defined as non-small-cell lung cancers (NSCLC) located at the superior sulcus with involvement of structures of the apical chest wall above the second

Accepted for publication January 5, 2016. Correspondence: Cornelis G Vos, MD, PhD; ncgvos@gmail.com. Disclosures: The authors report no disclosures or conflicts of interest. JCSO 2016;14:107-111. @2016 Frontline Medical Communications. doi: 10.12788/jicso.0233. 
rib. ${ }^{2,4}$ Eligibility for trimodality treatment was discussed in a multidisciplinary thoracic oncology meeting for all patients. Patients were excluded if they had pre-existing impaired function of the upper extremity from causes not related to trimodality treatment (ie, cerebrovascular accident, trauma, brain metastasis).

\section{Trimodality treatment}

All of the patients received trimodality treatment as published before. ${ }^{3,5}$ In short, it consisted of concurrent chemoradiotherapy followed by surgery. Chemotherapy consisted of 1 cycle cisplatin $80 \mathrm{mg} / \mathrm{m}^{2}$ on day 1 and gemcitabine $1,250 \mathrm{mg} / \mathrm{m}^{2}$ on days 1 and 8 for squamous histologies. Pemetrexed $500 \mathrm{mg} / \mathrm{m}^{2}$ on day 1 , and 2 cycles of cisplatin $80 \mathrm{mg} / \mathrm{m}^{2}$ on day 1 and etoposide $100 \mathrm{mg} / \mathrm{m}^{2}$ on days 1-3 for 3 weeks were used for nonsquamous histologies. Involved-field radiotherapy (46-66 Gy in fractions of 2 Gy) was started on day 2 of the second cycle of chemotherapy in all patients.

If no progression and no distant metastasis occurred, then patients had surgery about 6 weeks after completion of induction therapy. All of the patients received physiotherapy for breathing and coughing without any focus on arm function. An upper-lobe resection with en bloc resection of the involved chest wall and whenever necessary the $\mathrm{C} 8$ or $\mathrm{T} 1$ root of the brachial plexus was performed. Hilar and mediastinal lymph node dissection and covering of the bronchial stump with a vascularized intercostal muscle flap was routinely performed. Generally, tumors were approached through a Shaw-Paulson incision, although anteriorly located tumors were resected through a hemiclamshell incision. ${ }^{6}$

\section{Assessment of arm and shoulder disability and QoL}

The patient's overall quality of life was assessed using the 36-item Short Form Health Survey (SF-36) questionnaire. The Dutch language version of the SF-36 questionnaire is validated for general population surveys as well as for patients with cancer. ${ }^{7}$ The SF-36 questionnaire evaluates the following domains: physical functioning, social role functioning, physical role functioning, emotional role functioning, mental health, vitality, pain, general health perception, and health change. The scores can range from $0-100$, with higher scores indicating a better quality of life and lower scores indicating impairments of daily functioning. The disabilities of the arm, shoulder, and hand (DASH) questionnaire was used to assess the subjective disability of arm and shoulder function. ${ }^{8}, 9$ The DASH questionnaire is frequently used in functional studies after limb surgery. ${ }^{7,10}$ A higher score (maximum 100) implies a higher subjective disability of arm and shoulder function. Both questionnaires were assessed at 0 months (ie, preoperatively, after completion of induction chemoradiotherapy

\begin{tabular}{|c|c|}
\hline Characteristic & n (\%) \\
\hline $\begin{array}{l}\text { Median age, y (range): } \\
54 \text { (32-71) }\end{array}$ & - \\
\hline \multicolumn{2}{|l|}{ Clinical tumor stage (cTNM 7) } \\
\hline $2 \mathrm{~B}$ & $9(45)$ \\
\hline $3 \mathrm{~A}$ & $8(40)$ \\
\hline $3 B$ & $2(10)$ \\
\hline 4 & $1(5)$ \\
\hline $\begin{array}{l}\text { Median radiation dose, Gy } \\
\text { (range): } 50(45-66)\end{array}$ & - \\
\hline \multicolumn{2}{|l|}{ T1 nerve root resection } \\
\hline Yes & $13(65)$ \\
\hline No & $7(35)$ \\
\hline \multicolumn{2}{|l|}{ Dominant side } \\
\hline Left & $3(15)$ \\
\hline Right & $17(85)$ \\
\hline \multicolumn{2}{|l|}{ Operated on dominant side? } \\
\hline Yes & $14(70)$ \\
\hline No & $6(30)$ \\
\hline \multicolumn{2}{|l|}{ Approach operative technique } \\
\hline Shaw-Paulson & $18(90)$ \\
\hline Hemiclamshell & $2(10)$ \\
\hline
\end{tabular}

on the day before the operation) and 3 and 12 months postoperatively.

\section{Data collection}

In the prospectively maintained thoracic surgery database patients age, sex, treatment details, dominant side, staging characteristics (cTNM, ypTNM 7th edition ${ }^{11}$ ), pathology results, surgical details, complications, and follow-up data were stored and collected for the analysis.

\section{Statistical analysis}

All statistical analyses were performed using the statistical software package SPSS, version 17.0 (SPSS Inc, Chicago, IL, US). The scores of the DASH and SF-36 questionnaires assessed on the 3 time points (preoperatively, and 3 and 12 months postoperatively) were compared using the Wilcoxon signed rank test. In addition, a comparison was made between the scores of patients treated on their dominant side and of those treated on their nondominant side. A third analysis was performed, comparing scores of patients in whom T1 nerve root was resected to those in whom the T1 nerve root could be spared. For statistical evaluation of the latter 2 comparisons, the Mann-Whitney $\mathrm{U}$ test was used. Correlations were investigated using the Spearmans correlation coefficient. Missing data were left out of the analysis. Statistical significance was defined as $P<.05$. 
TABLE 2 DASH and SF-36 scores at 0 months (preoperatively) and 3 and 12 months postoperatively

\begin{tabular}{|c|c|c|c|}
\hline \multirow[b]{2}{*}{ Questionnaire } & \multicolumn{3}{|c|}{ Mean score (SD) [P value] } \\
\hline & 0 months $^{a}(n=16)$ & 3 months $^{\text {bc }}(n=17)$ & 12 months $^{\text {bd }}(n=18)$ \\
\hline DASH & $21(16)$ & $39(18)[.024]$ & 37 (18) [.011] \\
\hline \multicolumn{4}{|l|}{ SF-36 } \\
\hline Physical functioning & $71(21)$ & 61 (15) [ns] & $55(20)[.020]$ \\
\hline Social functioning & $63(29)$ & $65(33)[\mathrm{ns}]$ & $65(29)[\mathrm{ns}]$ \\
\hline Physical role functioning & $34(43)$ & 1 (6) [.041] & 21 (29) [ns] \\
\hline Emotional role functioning & $58(46)$ & $63(45)[n s]$ & $56(44)[n s]$ \\
\hline Mental health & $67(23)$ & $68(24)[n s]$ & $71(20)[n s]$ \\
\hline Vitality & $61(20)$ & 50 (19) [ns] & 57 (17) [ns] \\
\hline Pain & $69(16)$ & $44(22)[.006]$ & $54(20)[.019]$ \\
\hline General health perception & $50(16)$ & $54(20)[n s]$ & $48(24)[n s]$ \\
\hline Health change & $39(33)$ & $46(41)[n s]$ & $65(35)[n s]$ \\
\hline
\end{tabular}

DASH, disabilities of the arm and shoulder; ns, not significant (Wilcoxon signed rank test); SF-36, 36-item Short Form Health Survey

${ }^{a} 0$ months $=$ preoperatively. ${ }^{b} 3$ months and 12 months postoperatively. ${ }^{c} P$ values for comparison between 3 months and 0 months. ${ }^{\mathrm{d}} P$ values for comparison between 12 months and 0 months.

\section{Results}

\section{Patient characteristics}

Between April 1, 2010 and October 31, 2012, 22 patients received trimodality treatment for a SST in our center. Informed consent was obtained from 20 patients to participate in the study (Table 1). Four patients returned incomplete preoperative questionnaires, and 1 did not submit responses for 3-month postoperative questionnaire. During the follow-up period, 2 patients died within 3 months of surgery, leaving 18 patients who could be analyzed. All 18 completed the follow-up of 12 months.

\section{Treatment results}

A radical resection (R0) was performed in 19 of 20 patients (95\%). In 9 patients (45\%), a complete pathological response (no remaining vital tumor cells) was seen in the resection specimen. In 4 patients (20\%), more than $10 \%$ vital tumor cells were found, and the remaining 7 patients had fewer than $10 \%$ vital tumor cells remaining. Two patients developed brain metastasis at 3 and 8 months follow-up, respectively. In this cohort, there was no local recurrence within 12 months of follow-up. Two patients died within 3 months of surgery due to progression of disease (brain metastasis). At the end of the follow-up (12 months), 18 of 20 patients were alive.

\section{Arm and shoulder disability and quality of life}

Patients experienced a significantly higher subjective disability in arm and shoulder function at both 3 months $(P=.024)$ and 12 months $(P=.011)$ of follow-up com- pared with the preoperative measurement (Table 2). Patients scored significantly lower for the SF-36 domain physical functioning at 12 months $(P=.020)$ but not at 3 months. Scores for physical role functioning significantly deteriorated at 3 months $(P=.041)$, but at 12 months scores improved and the difference compared with the preoperative (0 months) score was no longer statistically significant. Patients reported significantly more pain at 3 months $(P=.006)$ and although pain scores improved, they still were significantly lower at 12 months $(P=.019)$ compared with 0 months. For the remaining domains (social functioning, emotional role functioning, mental health, vitality, general health perception, and health change) no statistically significant differences were found at the different time points.

\section{Subgroup analyses}

Subgroup analysis of DASH and SF-36 scores for patients with and without $\mathrm{T} 1$ nerve root resection failed to detect any significant difference except for the SF-36 domain health change at 3 months, where patients in whom the T1 nerve root was resected had significantly lower scores $(P=.037)$. After 1 year, this difference disappeared. No statistically significant differences were found for the DASH scores and scores on all SF-36 domains between patients who were operated on on their dominant side and those treated on their nondominant side. There were no correlations between the quality of life scores (SF-36 and DASH) and the dose of radiation, sex, age at the time of diagnosis, and the operative approach. 


\section{Discussion}

Arm and shoulder function and quality of life are negatively influenced by trimodality treatment for superior sulcus tumors. Patients reported increased subjective arm and shoulder function disability at 3 and 12 months postoperatively. The experienced physical functioning 1 year after resection was also worse. However, although the physical role functioning was significantly lower 3 months postoperatively, it returned to baseline (ie, to the first, preoperative measurement $[0$ months $]$ ) functioning after 1 year of follow-up. Patients reported significantly more pain 3 months postoperatively, and although pain scores improved thereafter,there was still significantly more pain reported compared with baseline. It is possible that pain scores could further improve with longer follow-up, but that is unclear at this time.

The influence of resection of the T1 nerve root seemed to be mild. Although patients initially reported a greater deterioration in their general health compared with those in whom the T1 nerve root could be spared, the differences disappeared after 1 year. Possible explanations could be that chemoradiotherapy might have a larger impact on arm function than surgery does; most of the function of the $\mathrm{T} 1$ branch might already be lost preoperatively because of compression or invasion by the tumor; and due to the subjective nature of the questionnaires, respondents might have considered their function satisfactory when taking into account that they are surviving a possibly lethal disease.

No correlations were found between the subjective arm and shoulder function and reported quality of life and treatment on the dominant side, radiotherapy dose, operative approach, age, and sex. These findings are in accordance with the results of our previous report in which we investigated arm and shoulder function and quality of live in a retrospective cohort of patients. ${ }^{3}$

In SST patients, arm and shoulder function might be at risk because of several causes. On the one and, compression or invasion of vital structures by these tumors located in the cervicothoracic junction and detrimental effects of radiotherapy, systemic chemotherapy (neuropathy), and surgery can all damage the nerves, vessels, muscles, and other structures vital for arm and shoulder function. On the other hand, trimodality treatment can improve arm function and pain by alleviating compression through shrinkage or resection of the tumor. ${ }^{12}$ Our study investigated the results of all these factors together.

Previous studies have mentioned that resection of the T1 nerve root is generally well tolerated but resection of C8 leads to loss of function, although objective measurements were not reported. ${ }^{13-15}$ Davis and colleagues reported that resection of the $\mathrm{T} 1$ nerve root (with or without the $\mathrm{C} 8$ nerve root) causes significant loss of function and investi- gated sparing of the $\mathrm{T} 1$ nerve root in only 5 patients with promising results, but they did not compare those results with patients in whom the T1 nerve root was resected. ${ }^{16}$ Shahian and colleagues scored the functional outcome of long-term survivors after resection of Pancoast tumors as excellent, good, fair, or poor, without further defining the groups. ${ }^{17}$ They reported that shoulder function is generally excellent, but is dependent on aggressive physical therapy after resection or radiotherapy. ${ }^{17}$

The present study is the largest reported study focusing on arm and shoulder function and quality of life in superior sulcus tumor patients. However, we recognize some limitations of the study. We did not formally test the actual arm and shoulder function but assessed it using validated questionnaires. Therefore, this study reports only the subjective arm and shoulder function. In addition, we included patients referred for surgery after chemoradiotherapy was administered, and no information was available on functional status and quality of life before the start of this induction treatment. The numbers of patients investigated were small, warranting a careful interpretation of our findings because of the risk of bias. For example survivorship bias (patients who died might have had worse outcomes) and nonresponse bias (patients with worse outcomes might more likely refuse participation) cannot be ruled out. The risk of bias is even higher when subgroup analyses are performed. Investigating correlations and searching for prognostic factors is limited by the small dataset and the risk for confounders. Moreover, the collected dataset was too small to perform a multivariate analysis to identify prognostic factors for arm and shoulder function and quality of life. Therefore, we failed to identify factors that could be influenced to improve function and quality of life in these patients. Moreover, in our cohort no local recurrence occurred and only 2 patients developed metastatic disease during follow-up. Although recurrence and metastases are probably important determinants of functional outcome and quality of life, the data provided by this study was insufficient to address this issue. However, the currently available literature on this topic is scarce, and the presented data could inform superior sulcus tumors patients.

\section{Conclusion}

Subjective arm function and quality of life are reduced following trimodality treatment. Resection of the T1 nerve root has no significant long-term effect on the subjective arm function and quality of life.

\section{Acknowledgments}

The authors thank Tjeerd van der Ploeg for his contribution to the statistical analysis. 


\section{References}

1. Alberts WM; American College of Chest Physicians. Diagnosis and management of lung cancer executive summary: ACCP evidencebased clinical practice guidelines (2nd ed). Chest. 2007;132:1S-19S.

2. Rusch VW, Giroux DJ, Kraut MJ, et al. Induction chemoradiation and surgical resection for superior sulcus non-small-cell lung carcinomas: long-term results of Southwest Oncology Group Trial 9416 (Intergroup Trial 0160). J Clin Oncol. 2007;25:313-18.

3. Gorter RR, Vos CG, Halmans J, Hartemink KJ, Paul MA, Oosterhuis JW. Evaluation of arm function and quality of life after trimodality treatment for superior sulcus tumours. Interact Cardiovasc Thorac Surg. 2013;16:44-48.

4. Detterbeck FC. Changes in the treatment of Pancoast tumors. Ann Thorac Surg 2003;75:1990-7.

5. Vos CG, Hartemink KJ, Blaauwgeers JL, Oosterhuis JW, Senan S, Smit EF et al. Trimodality therapy for superior sulcus tumours: evolution and evaluation of a treatment protocol. Eur J Surg Oncol. 2013;39:197-203.

6. Grunenwald D, Spiaggari L, Girard P, Baldeyrou P. Transmanubrial approach to the thoracic inlet. J Thorac Cardiovasc Surg. 1997;113:958-959.

7. Aaronson NK, Muller M, Cohen PDA, et al. Translation, validation, and norming of the Dutch language version of the SF-36 Health Survey in community and chronic disease populations. J Clin Epidemiol. 1998;11:1055-1068.

8. Hudak P, Amadio PC, Bombardier C, and the Upper Extremity Collaborative Group. Development of an upper extremity outcome measure: The DASH (Disabilities of the Arm, Shoulder, and Hand). Am J Ind Med. 1996;29:602-608.

9. Beaton DE, Katz JN, Fossel AH, Wright JG, Tarasuk V, Bombardier C. Measuring the whole or the parts? Validity, reliability \& respon- siveness of the disabilities of the arm, shoulder, and hand outcome measure in different regions of the upper extremity. J Hand Ther. 2001;14:128-146.

10. Simmen BR, Angst F, Schwyzer HK, et al. A concept for comprehensively measuring health, function and quality of life following orthopaedic interventions of the upper extremity. Orthop Trauma Surg. 2009;129:113-118.

11. Vallières E, Shepherd FA, Crowley J, et al. The IASLC Lung Cancer Staging Project: proposals regarding the relevance of TNM in the pathologic staging of small cell lung cancer in the forthcoming (7th) edition of the TNM classification for lung cancer. J Thorac Oncol. 2009;4:1049-1059.

12. Komaki R, Roh J, Cox JD, Lopes de Conceicao A. Superior sulcus tumors: results of irradiation of 36 patients. Cancer. 1981;48:1563-1568.

13. Rusch VW. Management of Pancoast tumours. Lancet Oncol. 2006;7:997-1005.

14. Mazel C, Grunenwald D, Laundrin P, Marmorat JL. Radical excision in the management of the thoracic and cervicothoracic tumors involving the spine: results in a series of 36 cases. Spine. 2003;28:782-792.

15. Bilsky MH, Vitaz TW, Boland PJ, Bains MS, Rajaraman V, Rusch VW. Surgical treatment of superior sulcus tumors with spinal and brachial plexus involvement. J Neurosurg. 2002;97:301-309.

16. Davis GA, Knight S. Pancoast tumor resection with preservation of brachial plexus and hand function. Neurosurg Focus. 2007;22:E15.

17. Shahian DM, Neptune WB, Ellis FH. Pancoast tumors: improved survival with preoperative and postoperative radiotherapy. Ann Thorac Surg. 1987;43:32-38. 